\title{
Erbium-doped nanocrystalline silicon thin films produced by RF sputtering, annealing effect on the Er emission
}

\author{
$\underline{\text { M.F. Cerqueira }}{ }^{1}$, T. Monteiro ${ }^{2}$, M. J. Soares ${ }^{2}$, A. Kozanecki ${ }^{3}$, P. Alpuim ${ }^{1}$ and E. Alves ${ }^{4}$ \\ ${ }^{1}$ Departamento de Física, Universidade do Minho, Braga, Portugal. \\ ${ }^{2}$ Departamento de Física e I3N, Universidade de Aveiro, 3710-193 Aveiro, Portugal. \\ ${ }^{3}$ Institute of Physics, PAS, Warsaw, Poland \\ ${ }^{4}$ Instituto Tecnológico e Nuclear, EN 10, 2686-953 Sacavém, Portugal \\ Contact author: fcerqueira@ fisica.uminho.pt
}

In the present work, erbium doped nanocrystalline silicon thin films were produced by reactive magnetron sputtering on glass substrates under different conditions (substrate temperature and Er content). The film structure was studied using Raman spectroscopy. The chemical composition was determined using the RBS technique.

All the samples show sharp ${ }^{4} \mathrm{I}_{13 / 2} \rightarrow{ }^{4} \mathrm{I}_{15 / 2}$ intra- $4 f_{11}$ emission of $\mathrm{Er}^{3+}$ related centres with its maximum positioned at the $1.54 \mu \mathrm{m}$. However, the intensity of this transition (strongly dependent on the chemical composition of the matrix where the nanocrystals are embedded in and also on the structure of the matrix) changes after thermal annealing treatment. For the less crystalline samples our results show an increase of the $\mathrm{Er}^{3+} \mathrm{PL}$ intensity and for the highly crystalline ones the Er emission vanishes even at low temperature. This behavior was studied and explained in this work, on the basis of energy transfer between $\mathrm{Si}$ and Er ions.

\section{Introduction}

The achievement of efficient room-temperature light emission from $\mathrm{Si}$ is a crucial step toward the fully integrated Si-based optoelectronics. Erbium doped crystalline silicon (c$\mathrm{Si}: \mathrm{Er})$ is a strong candidate for the fabrication of emitters and detectors to be used in optical telecommunication systems due to its intraionic luminescence line at $1.54 \mu \mathrm{m}$. However, the severe thermal quenching of the photoluminescence observed in c-Si:Er, makes the effectiveness of this system questionable [1]. The situation may be improved by the incorporation of Er ions in hydrogenated nanocrystalline silicon (nc-Si:Er) [2]. 
Nanocrystalline silicon thin films consist of small $\mathrm{Si}$ crystals embedded in an hydrogenated amorphous matrix $(\mathrm{a}-\mathrm{Si}: \mathrm{H})$. The silicon nanocrystal size is typically in the range form 3 to $8 \mathrm{~nm}$. Silicon nanocrystals (NCs) may act as the excitation species for Er ions, though the confinement of NCs in the amorphous Si matrix is hard to realize.

In this contribution we investigate thin films with this type of structure, studying Errelated PL dependence on the film structure, annealing and chemical composition of the matrix. Erbium-doped nanocrystalline silicon thin films were deposited by the reactive magnetron sputtering tech-nique, using a c-Si target covered with pieces of metallic Er. It is shown that it is possible to produce by this method Er-doped nc-Si:H thin films emitting at room temperature. The advantage of these films, compared to the more common $\mathrm{SiO} 2$ structures containing $\mathrm{Si} \mathrm{NCs}$ is their relatively high electrical conductivity, which makes the material attractive for device applications.

\section{Experimental}

Erbium doped nanocrystalline silicon (nc-Si:Er) thin films were deposited by radio frequency (RF) plasma assisted reactive magnetron sputtering in an $\mathrm{Ar} / \mathrm{H} 2$ atmosphere on ordinary glass substrates. Samples with different structural parameters (overall crystallinity, CR; crystal size, D, and matrix composition), were obtained by varying the deposition parameters. The fixed deposition parameters are the RF power $(80 \mathrm{~W})$ and the hydrogen fraction defined as $\mathrm{RH}=\mathrm{pH}_{2} /\left(\mathrm{pH}_{2}+\mathrm{pAr}\right)=0.63$ where the $\mathrm{pH}_{2}$ and $\mathrm{pAr}$ are the partial pressure of $\mathrm{H} 2$ and Ar respectively. The substrate temperature was $50^{\circ} \mathrm{C}$ for $\mathrm{Er} 34$ sample, $150^{\circ} \mathrm{C}$ for $\mathrm{P} 13$ sample and $300^{\circ} \mathrm{C}$ for the others depositions. The target used was a c-Si of high purity (99.99\%). The erbium was placed in a low erosion area on the silicon target, in order to keep the moderate rate of Er doping. The substrate-target distance was fixed at $55 \mathrm{~mm}$. The chemical composition was determined by Rutherford Backscattering Spectroscopy (RBS) and Elastic Recoil Detection (ERD) technique. The structural characterisation was done by micro-Raman spectroscopy under excitation with the $514.5 \mathrm{~nm}$ line of an $\mathrm{Ar}^{+}$laser using a Jobin Yvon triple monochromator. With the 488 $\mathrm{nm}$ and $514.5 \mathrm{~nm}$ lines of an $\mathrm{Ar}^{+}$laser as excitation sources, photoluminescence measurements in the infrared spectral region have been performed in the temperature range of 70-300 K using a Brucker 66V Fourier-transform spectrometer. The signal was 
detected with a North-Coast EO-817 liquid nitrogen cooled germanium detector. The structural characterization as well as the erbium emission was evaluated prior and after the annealing treatment $\left(400^{\circ} \mathrm{C}\right.$ for $\left.2 \mathrm{~h}\right)$.

\section{Results and discussion}

The chemical composition was determined by combining the Rutherford Backscattering Spectroscopy (RBS) and Elastic Recoil Detection (ERD) techniques (see Table 1). Some deposited films are characterized by low oxygen content and large hydrogen content (samples Er34, P13 and P17), whereas P25 sample has large oxygen content when compared with their hydrogen content.

The average nanocrystal size and the crystalline phase fraction, presented in Table 1, were estimated from the Raman spectra by fitting them in the framework of the Campbell-Fauchet model [3]. These structural parameters were confirmed using the standard procedure based on transmission electron microscopy (TEM) and X-ray diffraction studies as described in ref [4].

Table 1 - Atomic concentration and structural parameters: DR - average crystal size by Raman; CR- crystalline volume fraction by Raman; d-film thickness.

\begin{tabular}{|c|c|c|c|c|c|c|c|}
\hline Sample & $\begin{array}{c}\mathrm{Er} \\
(\mathrm{at} \%)\end{array}$ & $\begin{array}{c}\mathrm{Si} \\
(\mathrm{at} \%)\end{array}$ & $\begin{array}{c}\mathrm{O} \\
(\mathrm{at} \%)\end{array}$ & $\begin{array}{c}\mathrm{H} \\
(\mathrm{at} \%)\end{array}$ & $\begin{array}{c}\mathrm{D}_{\mathrm{R}} \\
(\mathrm{nm})\end{array}$ & $\begin{array}{c}\mathrm{C}_{\mathrm{R}} \\
(\%)\end{array}$ & $\mathrm{d}(\mathrm{nm})$ \\
\hline Er34 & 0.03 & 72 & $<4$ & 28 & $<3$ & $34^{*}$ & 1900 \\
\hline P13 & 0.55 & 70 & 9 & 18 & $<3$ & $34^{*}$ & 760 \\
\hline P17 & 0.6 & 65 & 10 & 25 & $>7$ & 52 & 1260 \\
\hline P25 & 0.89 & 74 & 16 & 9 & $>7$ & 61 & 480 \\
\hline
\end{tabular}

*estimated from TEM images

Figure 1 shows the Raman spectra of the as-deposited films. The presence of silicon nanocrystals is identified by the appearance of the TO vibrational mode characteristic of c-Si (approximately at $520 \mathrm{~cm}^{-1}$ ). The presence of a-Si:H is confirmed by a broad band at around $480 \mathrm{~cm}-1$. It is seen that all the produced samples show the presence of a silicon amorphous phase. The crystalline volume fraction of the films (for the fixed RF power) 
increases with the in-crease of the substrate temperature, from $34 \%$ to $61 \%$ at $50^{\circ} \mathrm{C}$ and $300^{\circ} \mathrm{C}$, respectively.

Although samples grown at substrate temperature between $50^{\circ} \mathrm{C}$ and $150^{\circ} \mathrm{C}$ look like amorphous by Raman analysis, the Er34 sample, has been extensively characterized by transmission electron microscopy (TEM) (see ref [5]) shows a volume fraction of $34 \%$ of silicon nanocrystallites with a size $<3 \mathrm{~nm}$ embedded in an a-Si:H matrix.

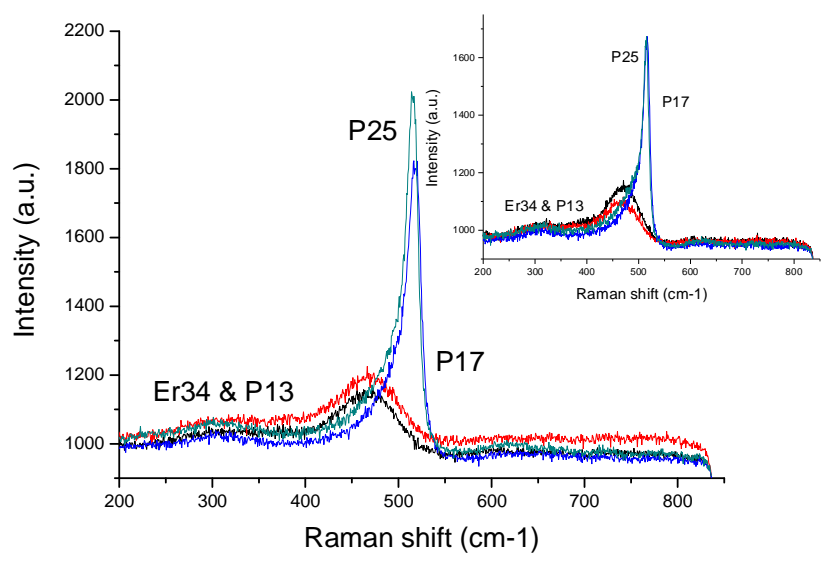

Figure 1 - Raman spectra of studied films showing the amorphous and crystalline components. Inset shows the Raman spectra of the samples annealed at $400^{\circ} \mathrm{C}$.

According to the Raman analysis, the as-grown erbium-doped silicon samples produced by RF sputtering can be separated into two groups, namely one group of samples having Si crystal sizes lower than $3 \mathrm{~nm}$ (Er34 and P13) and the other group having larger silicon crystals size (P17 and P25 samples). The estimated values, based on Raman spectra, of the crystalline volume fraction, $\mathrm{CR}$, and of the crystallite size, DR, are given in Table 1.

With the aim of investigating how the different micro-structures $(\mathrm{D}, \mathrm{C}$ and chemical matrix composition) influence the $\mathrm{Er}^{3+}$ photoluminescence (PL), the room temperature PL spectra of the as-grown samples are compared in Fig. 2. All the spectra are characterised by the presence of the $1.54 \square \mathrm{m}$ peak originated by interatomic ${ }^{4} \mathrm{I}_{13 / 2} \rightarrow$ ${ }^{4} \mathrm{I}_{15 / 2}$ transition of $\mathrm{Er}^{3+}$ ions. The $\mathrm{Er}^{3+}$ luminescence is more in-tense for the samples having small sized Si nanocrystals $(<3 \mathrm{~nm})$ than for those having larger sized NCs $(>7$ $\mathrm{nm}$ ), in agreement with other groups [6]. PL was excited off-resonance, so to have intense PL at room temperature two factors are necessary: efficient excitation and 
reduced quenching. It is well known that $\mathrm{Er}$ in a-Si:H shows much less thermal quenching than $\mathrm{Er}$ in $\mathrm{c}-\mathrm{Si}[7]$ and this is the most obvious explanation. However, since in all the samples there exist Si NCs, we can not exclude another mechanism, that means the $\mathrm{Si}$ size effect. Interestingly, the most intense $\mathrm{Er}^{3+} \mathrm{PL}$ peak are recorded for samples with quite different erbium content (Er34 having 0.03 at.\% and P13 having 0.55 at.\%). From these facts we can infer that (i) Er concentration of 0.03 at\% is enough to obtaining the characteristic Er3+ emission and (ii) 0.55 at.\% is not in excess for getting the Er emission, this means that, in this concentration range, erbium does not form Er metallic clusters in the films or non emitting components...

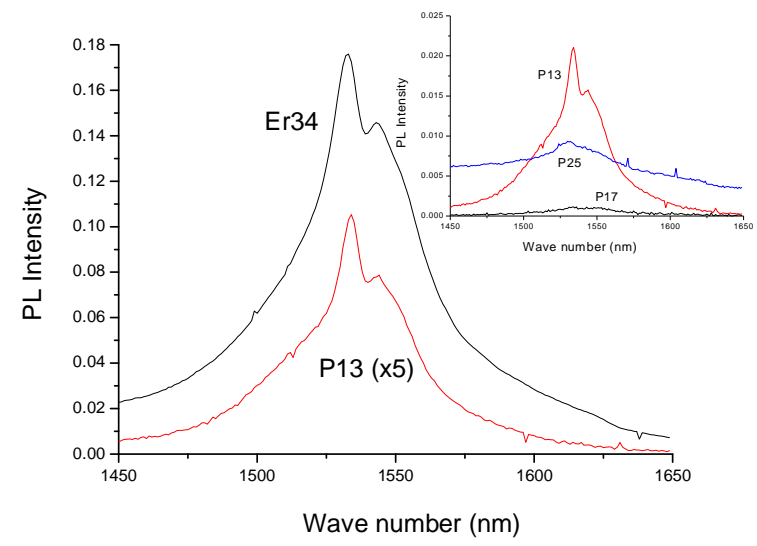

Figure 2 - Room temperature (RT) ${ }^{4} \mathrm{I}_{13 / 2} \rightarrow{ }^{4} \mathrm{I}_{15 / 2} \mathrm{Er}^{3+}$ luminescence spectra obtained with the $514.5 \mathrm{~nm}$ laser line excitation for the as-deposited samples with low-crystalline fraction. Inset: RT PL for samples with high-crystalline fraction (P13 is for comparison)

Furthermore, since films Er34 and P13 (with D $<3 \mathrm{~nm}$ and low crystalline volume fraction) have very different hydrogen and oxygen content and show the highest $\mathrm{Er}^{3+} \mathrm{PL}$ intensity, we can infer that the matrix chemical composition is not the key factor controlling the $\mathrm{Er}^{3+}$ emission. Moreover samples P17 and P25 show almost no $\mathrm{Er}^{3+} \mathrm{PL}$ having also very different chemical composition. We conclude that in a mixed phase material (a-Si:H and nc-Si) small Si nanocrystals are more favourable than large Si NCs to the energy transfer to the erbium ions. Indeed, this result is in agreement with Franzo et al [8] that have re-ported that for Si-rich material, the only crucial point is the presence 
of a high density of small Si clusters that can act as both the absorbing medium and sensitizers for Er.

In order to clearly identify this crystal size effect on the excitation of Er ions, the films were thermal annealed in air for $2 \mathrm{~h}$ at $400^{\circ} \mathrm{C}$. Figure 3 depicts the room temperature photoluminescence spectra of the annealed samples showing the $\mathrm{Er}^{3+}$ luminescence around $1.54 \mu \mathrm{m}$. As obtained for the as-deposited samples, the low-crystalline fraction samples (Er34 and P13) show stronger Er3+ luminescence than the samples with higher crystalline content (P17 and P25), for which the intraionic PL is nearly absent. Interesting are the results observed for the Er34 sample, with small sizes of the nanocrystals, which shows, again, the highest intensity.

The Raman spectra of the annealed films are similar to those obtained before annealing (Inset of Fig.1). Thus, the thermal annealing treatment had no effect on the sample structure, i.e. the $400^{\circ} \mathrm{C}$ annealing treatment did not induce sample crystallization. It is known that for a-Si:H thin films annealing at temperatures higher than $300-350^{\circ} \mathrm{C}$ induce the outdiffusion of hydrogen and consequent increase of structural disorder [9]. The hydrogen outdiffusion and the consequent increase of structural disorder are then expected for the annealed samples in the present work. How-ever no changes in the FWHM values of the Raman peaks have been observed. Therefore neither crystallization nor increase of structural disorder did occur as a consequence of the annealing treatment. However the intensity of $\mathrm{Er}^{3+}$ still increases for the low-crystalline samples and vanished for the high-crystalline ones, after annealing.

However, at this annealing temperature, it is possible that a partial atomic reordering occurs, not detected by Raman [10] possibly accompanied by the diffusion of light impurities in the Si-network. Since there was no observed hydrogen exodiffusion (no changes in the FWHM values of the Raman peaks), the effect of the annealing on the low-crystalline samples (having hydrogen content between 17 and 27 at.\%) could be a reduction of the number of defects through passivation with hydrogen. Consequently a reduction of the number of non-radiative pathways for re-combination of electron-hole pairs occurs and so the $\mathrm{Er}^{3+} \mathrm{PL}$ increases. This process of network local reconstruction can also lead to the activation of the charge-transfer dopants since it is assisted by mobile light atoms (e.g. hydrogen or oxygen) in the Si network $[11,12]$. For the high-crystalline 
samples, the annealing effect was to decrease $\mathrm{Er}^{3+} \mathrm{PL}$. It is known that hydrogen exodiffusion curves from nc-Si:H show a low-temperature peak at around $250^{\circ} \mathrm{C}$, a lower temperature the one observed for the corresponding peak in a-Si:H films [13]. Probably for the high CR samples with a large number of grain boundaries, the atomic hydrogen evolves from the silicon network, leaving behind many dangling bonds (DB) at the grain boundaries. These DB, will act as non-radiative recombination centres that will hinder energy transfer to the emitting centre.

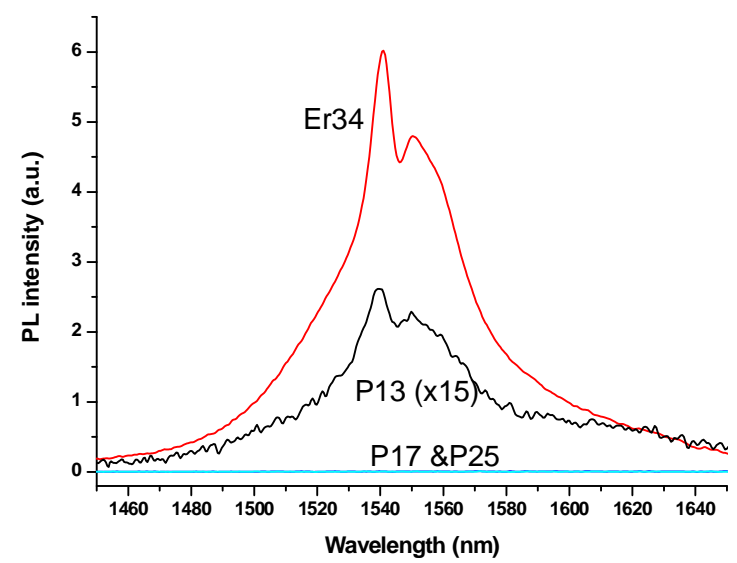

Figure 3 - Room temperature (RT) ${ }^{4} \mathrm{I}_{13 / 2} \rightarrow{ }^{4} \mathrm{I}_{15 / 2} \mathrm{Er}^{3+}$ luminescence spectra obtained with the $488 \mathrm{~nm}$ laser line excitation for the annealed samples.

For the better understanding the annealing effect, low temperature PL measurements of the annealed samples were performed. Figure 4 shows the PL spectra recorded at $70 \mathrm{~K}$, for the emitting samples. Once again, only the samples with lower crystalline fraction reveal intraionic PL. Even when the temperature is cold down the $\mathrm{Er}^{3+} \mathrm{PL}$ is absent for the highest crystalline samples, although they have erbium content higher than 0.03 at\% and the hydrogen content although lower than for Er34 sample is still high (25 at\% for P17 and 9 at.\% for P25 sample) meaning that the energy transfer becomes even less efficient after annealing for these samples.

For samples with the lower crystalline fraction (Er34 and P13) the $\mathrm{Er}^{3+} \mathrm{PL}$ intensity at RT is nearly $50 \%$ of its initial value at $70 \mathrm{~K}$ for both samples which suggests similar extinction processes. 


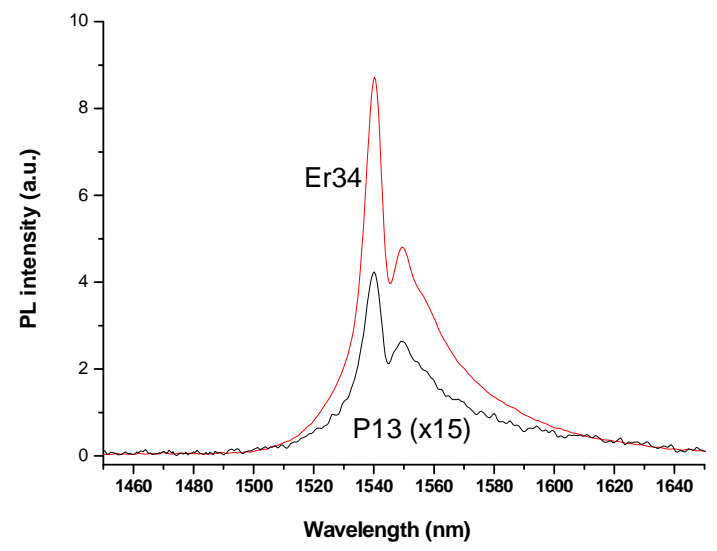

Figure $4-70 \mathrm{~K}^{4} \mathrm{I}_{13 / 2} \rightarrow{ }^{4} \mathrm{I}_{15 / 2} \mathrm{Er}^{3+}$ luminescence spectra obtained with the $488 \mathrm{~nm}$ laser line excitation for the annealed samples.

A more accurate analysis was performed for sample Er34 as shown in Figure 5. Between $70 \mathrm{~K}$ and $180 \mathrm{~K}$ the $\mathrm{Er}^{3+}$ integrated intensity is well account by a thermal population described by an activation energy of $5 \mathrm{meV}$, fol-lowed, for higher temperatures of nonradiative de-excitation processes that can be well fitted to an activation energy of 200meV. The Er34 sample presents the highest PL intensity. As both the Er34 and P13 samples do not differ in microstructure but in matrix chemical composition (sample Er34 having much more hydrogen than P13), we suggest that its much higher PL intensity could be deter-mined by the chemical composition of the Si matrix.

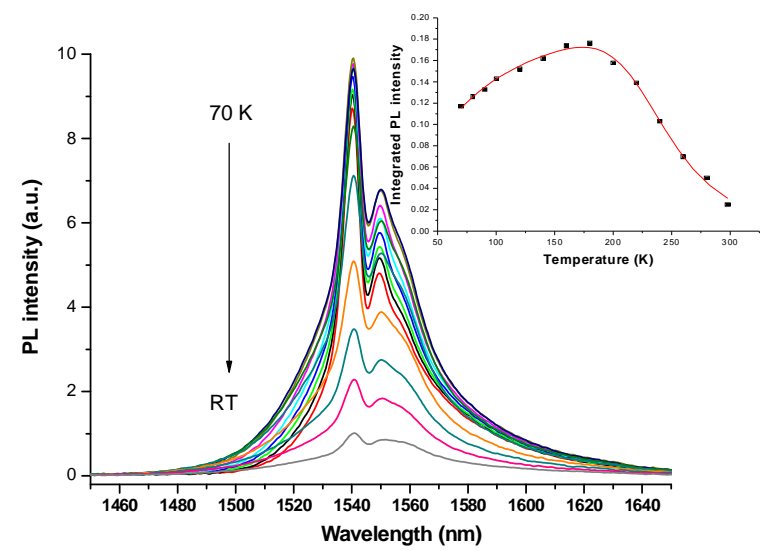

Figure 5 - Temperature dependence of the ${ }^{4} \mathrm{I}_{13 / 2} \rightarrow{ }^{4} \mathrm{I}_{15 / 2} \mathrm{Er}^{3+}$ luminescence spectra obtained with the $488 \mathrm{~nm}$ laser line excitation for the Er34 sample. Inset: Evolution of the integrated PL intensity with temperature. 


\section{Conclusions}

Er-doped nc-Si:Er:H films were deposited by co-sputtering. After deposition the films were annealed and investigated through Raman spectroscopy and PL measurements. According to the experimental results, annealing at $400^{\circ} \mathrm{C}$ does not cause important structural changes. However, a considerable change on the Er-related PL intensity at $1540 \mathrm{~nm}$ was verified: an enhancement of the PL for the less crystalline films and a suppression of the PL for the highly crystalline films. From the results of the present work, it can be conclude that in the case of mixed phase (amorphous matrix with Si NCs embedded in): (i) there exist a higher excitation efficiency of Er ions for samples having small nanocrystals than bigger ones; (ii) the enhancement of the luminescence efficiency, after annealing, in the less crystalline samples seems to be deter-mined by chemical aspects of the Si matrix, mainly hydrogen content; (iii) the practically vanished Er3+ luminescence for the highly crystalline samples suggest that it is consequence of the reduced excitation efficiency of Er ions due to the presence of a large number of big crystals.

\section{References}

[1] Y. Kanzawa, T. Kakevka, M. Fujii, S. Hayashi, K. Yamamoto, Solid State Commun. 102, 533 (1997).

[2] "Silicon-Based Optoelectronics", ed. by S. Coffa and L. Tsy-beskov, MRS Bulletin 23, 16 (1998).

[3] I. H. Campbell and P. M. Fauchet, Solid State Commun. 58, 739 (1986).

[4] M.F. Cerqueira, J.A. Ferreira, G. Adriaenssens, Thin Solid Films 370, 128 (2000).

[5] M. Losurdo, M.M. Giangregorio, P. Capezzuto, G. Bruno, M.F. Cerqueira, E. Alves, M. Stepikhova, Appl. Phys. Lett. 83, 2993 (2002).

[6] N. Park, T. Kim, S.H. Kim, G.Y. Sung, K.S. Cho, J.H. Shin, B. Kim, S. Park, J. Lee, M. Nastasi, Thin Solid Films 475, 231 (2005).

[7] T. Oestereich, C. Swiatowski, I. Broser, Appl. Phys. Lett. 56, 446 (1990)

[8] G. Franzo, S. Boninelli, D. Pacifici, and F. Priolo, F. Iacona and C. Bongiorno, Appl. Phys. Lett 82, 3871 (2003)

[9] A. R. Zanatta and F. L. Freire, Jr., Physical Review B 62, 2016 ( 2000).

[10] A. R. Zanatta, M. Mulato, and I. Chambouleyron, J. Appl. Phys. 84, 5184 (1998).

[11] L.R. Tessler, C. Piamontezem, M.C. Martins Alves, H. To-lentino, J. Non-Cryst. Solids 266-269, 598 (2000). 
[12] A. Kozanecki, D. Kuritsyn, H. Przybyliska, W. Jantsch, Physica B: Cond. Matter 308-310, 354 (2001)

[13] L. Raniero, I. Ferreira, L. Pereira, H. Águas, E. Fortunato, R. Martins, J. Non-Cryst. Solids 352, 1945 (2006) 